\title{
Variant Maps of Elementary Equations
}

\author{
Jeffrey Zheng
}

\begin{abstract}
Using four measures in Type B, there are 11 invariant expressions to form elementary equations of variant measurement. In this chapter, two invariant expressions are selected to illustrate sample procedures from elementary equations to relevant variant maps. Using various projections and multiple levels of representations, complicated binomial coefficients and their variations are illustrated under various conditions. Using multinomial coefficients, multiple viewpoints are used for references. Due to this type of variation framework contains rich structures, further explorations are required from multiple levels on both theoretical foundation and practical applications.
\end{abstract}

Keywords Variant measurement $\cdot$ Elementary equation · Variant map Multinomial coefficient $\cdot$ Coefficient array

\section{Introduction}

Variant construction starts from $n 0-1$ variables to form $2^{n}$ states and $2^{2^{n}}$ functions, via vector permutation and complement operations on state space to establish a variant logic framework to contain $2^{n} ! \times 2^{2^{n}}$ configurations as a variation space. Variant measurement acts as a core of quantitative measurement, starting from $m 0-1$ variables to explore relevant clustering conditions on $2^{m}$ states. Since this type of variations has a close relationship to partition and recombination using binomial and multinomial coefficients under identically combinatorial expressions. Apply-

This work was supported by the Key Project on Electric Information and Next Generation IT Technology of Yunnan (2018ZI002), NSF of China (61362014), Yunnan Advanced Overseas Scholar Project.

J. Zheng (凶)

Key Laboratory of Quantum Information of Yunnan, Yunnan University, Kunming, China e-mail: conjugatelogic@yahoo.com

J. Zheng

Key Laboratory of Software Engineering of Yunnan, Yunnan University, Kunming, China

(C) The Author(s) 2019

J. Zheng (ed.), Variant Construction from Theoretical Foundation to Applications, https://doi.org/10.1007/978-981-13-2282-2_6 
ing the results in Chapter "Elementary Equations of Variant Measurement", Type B measures are composed of 11 nontrivial invariants. Two invariants are selected in this chapter, their different partition properties are illustrated to use coefficients on 2D and 3D distributions. Variant maps are generated from coefficient arrays as samples.

\section{Measures and Maps}

Two combinatorial invariants are selected: $\{m-p\}\{p\}$ and $\{2 q\}\{m-2 q\}$. Different distributions on their coefficients are explored.

\subsection{Case $1 .\{m-p\}\{p\}$}

For $\{m-p\}\{p\}$ formula, relevant equation is

$$
\left(\begin{array}{l}
m \\
p
\end{array}\right)=\sum_{k=0}^{p}\left(\begin{array}{c}
m-p \\
k
\end{array}\right)\left(\begin{array}{l}
p \\
k
\end{array}\right)
$$

A binomial coefficient is separated by sum of $(p+1)$ pairs of binomial coefficient products. For a selected value $p$, coefficients $\left\{\left(\begin{array}{c}m-p \\ k\end{array}\right)\left(\begin{array}{l}p \\ k\end{array}\right)\right\}, 0 \leq k \leq p$ are arranged in a linear order.

This property is true for all $p$ values. A special three tuple structure $(m, p, k)$ has $1-1$ correspondence with a coefficient $f(m, p, k)=\left(\begin{array}{c}m-p \\ k\end{array}\right)\left(\begin{array}{l}p \\ k\end{array}\right)$. While $m$ value increased, coefficient array will be increased as a 3D rectangular steps, each $m$ value has a $(m+1)^{2}$ region.

The nontrivial coefficients are distributed as a triangle. $\operatorname{Let} F(m, p)=\sum_{\forall k}$ $f(m, p, k), 0 \leq p \leq m$ and $G(m, k)=\sum_{\forall p} f(m, p, k), 0 \leq k \leq m$, two projections $\{F(m, p), G(m, k)\}$ can be projected. Coefficients and relevant four maps are shown in Fig. 1.

Lemma 1 For $\{m-p\}\{p\}$ equation, coefficients are distributed in $(m+1)^{2}$ and all nontrivial coefficients are clustered in 1/4 region and 3/4 regions has coefficient 0 .

\subsection{Case 2. $\{2 q\}\{m-2 q\}$}

Briefly $\{m-p\}\{p\}$ and $\{2 q\}\{m-2 q\}$ are simple invariants. For $\{2 q\}\{m-2 q\}$ invariant, it has the following equation. 


\begin{tabular}{|c|c|c|c|c|c|c|c|c|c|c|c|}
\hline$f(10, p, k)=()$. & $\begin{array}{lll}0 & 1\end{array}$ & 2 & 3 & 4 & 5 & 6 & 7 & 8 & 9 & & $G(10, k)=\sum_{\forall p}()$. \\
\hline 0 & $\begin{array}{ll}1 & 1\end{array}$ & 1 & 1 & 1 & 1 & 1 & 1 & 1 & 1 & & 11 \\
\hline 1 & 9 & 16 & 21 & 24 & 25 & 24 & 21 & 16 & 9 & & 165 \\
\hline 2 & & 28 & 63 & 90 & 100 & 90 & 63 & 28 & & & 462 \\
\hline 3 & & & 35 & 80 & 100 & 80 & 35 & & & & 330 \\
\hline 4 & & & & 15 & 25 & 15 & & & & & 55 \\
\hline 5 & & & & & 1 & & & & & & 1 \\
\hline 6 & & & & & & & & & & & \\
\hline 7 & & & & & & & & & & & \\
\hline 8 & & & & & & & & & & & \\
\hline 9 & & & & & & & & & & & \\
\hline 10 & & & & & & & & & & & \\
\hline$k$ & & & & & & & & & & & \\
\hline$F(10, p)=\sum_{\forall k}()$. & 110 & 045 & 120 & 210 & 252 & 210 & 120 & 45 & & & $\sum_{\forall p, k}()=1024=.2^{10}$ \\
\hline
\end{tabular}

(a)

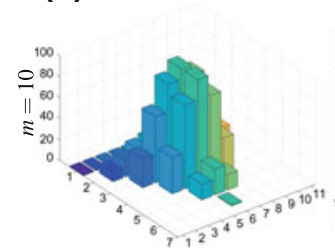

(b)

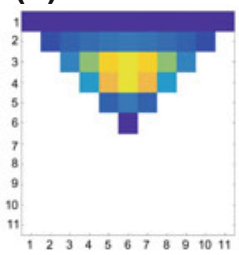

(c)

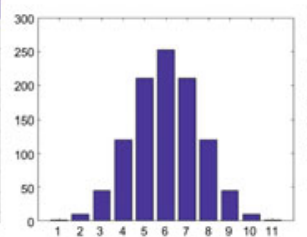

(d)

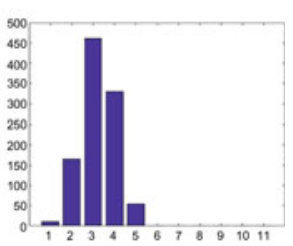

Fig. 1 One set of coefficients and its two projections in four maps (a)-(d); a 3D $f(10, p, k)$; b 2D $f(10, p, k)$; c $1 \mathrm{D} F(10, p)$; d $1 \mathrm{D} G(10, k)$

$$
\left(\begin{array}{c}
m \\
p
\end{array}\right)=\sum_{k=0}^{p}\left(\begin{array}{c}
2 q \\
k
\end{array}\right)\left(\begin{array}{c}
m-2 q \\
p-k
\end{array}\right)
$$

where $q$ is a free variable, $0 \leq q \leq\lfloor m / 2\rfloor$. Different from Case 1, this equation can determine $l$ floorm $/ 2\rfloor+1$ levels of coefficients according to different $q$ values selected to form a 3D coefficient structure.

Let $f(m, q, p . k)=\left(\begin{array}{c}2 q \\ k\end{array}\right)\left(\begin{array}{c}m-2 q \\ p-k\end{array}\right)$ under $0 \leq q \leq\lfloor m / 2\rfloor, 0 \leq k, p \leq m$ conditions, nontrivial coefficients are distributed in special shapes on multiple 2D regions.

Using color coding scheme, it is feasible to map coefficients into greyscale or color pixels as variant maps.

A binomial coefficient can be separated as sum of $(p+1)$ pairs of coefficient products $\left\{\left(\begin{array}{c}2 q \\ k\end{array}\right)\left(\begin{array}{c}m-2 q \\ p-k\end{array}\right)\right\}, 0 \leq k \leq p$ to be a linear order.

This type of property is true for all $p$ values, a special tuple of four parameters $(m, q, p, k)$ has $1-1$ correspondence with coefficient $\left(\begin{array}{c}2 q \\ k\end{array}\right)\left(\begin{array}{c}m-2 q \\ p-k\end{array}\right)$. Each selected $m$ value is corresponding to $(m+1)^{2} \times(\lfloor m / 2\rfloor+1)$ region to locate all coefficients.

Lemma 2 For $\{2 q\}\{m-2 q\}$ combinatorial invariant, all coefficients are restricted in $(m+1)^{2} \times(\lfloor m / 2\rfloor+1)$ region. 


\section{Visual Results}

It is convenient to use color coding to transfer each coefficient as a pixel in a variant map. Invariant coefficients provide ideal conditions for a practical measurement, it is feasible to check physical differences between an idea distribution and a practical measurement.

From a quantitative viewpoint, multinomial expressions provide proper basis on corresponding partitions to be a relative measurement in representation.

\subsection{Case 1. Maps}

Using $\left(\begin{array}{c}m \\ p\end{array}\right) \rightarrow\left\{\left(\begin{array}{c}m-p \\ k\end{array}\right)\left(\begin{array}{l}p \\ k\end{array}\right)\right\}$, three maps are shown in Fig. 1 as $2 \mathrm{D}$ coefficients, 3D histograms, and 2D projections on four parameters $m=\{10,11,15,16\}$, respectively.

\subsection{Case 2. Maps}

Different from Case 1 , each $m$ is associated with one 2D coefficient. In $\left(\begin{array}{c}m \\ p\end{array}\right) \rightarrow$ $\left\{\left(\begin{array}{c}2 q \\ k\end{array}\right)\left(\begin{array}{c}m-2 q \\ p-k\end{array}\right)\right\}$ conditions, each $q$ selection determines a $2 \mathrm{D}$ array of coefficients. Under $0 \leq q \leq\lfloor m / 2\rfloor$ conditions, $\lfloor m / 2\rfloor+1$ levels are required. For $m=10$, it is necessary to have 6 levels.

To observe global properties, a 3D color map is shown in Fig. 3 to illustrate 3D coefficients under color coding.

\section{Result Analysis}

In maps of Figs. 1, 2, and 3, it is convenient to see variant maps transformed from elementary equations. From a certain viewpoint, $\{m-p\}\{p\}$ coefficients have symmetric properties on horizontal direction on $p: m-p$ with reflective properties. Nontrivial coefficients are located in $1 / 4$ region of $(m+1)^{2}$ square. An isosceles triangle is composed of all nontrivial coefficients. Selecting any $m$, there is only one $2 \mathrm{D}$ coefficient associated with to be a unified distribution.

$\{2 q\}\{m-2 q\}$ coefficients are corresponding to multiple $2 \mathrm{D}$ distributions under various $q$ values. While $q=0$, each nontrivial coefficient is located on diagonal position of $p=k$ and each coefficient is a $\left(\begin{array}{c}2 q \\ k\end{array}\right)\left(\begin{array}{c}m-2 q \\ p-k\end{array}\right)$ equation. In $0 \leq q \leq 5$ conditions, 2D coefficient matrices are shown in six groups of $\{0: 10,2: 8,4: 6,6$ : 


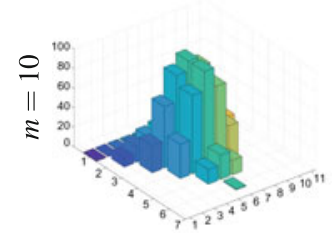

$\left(a_{1}\right)$

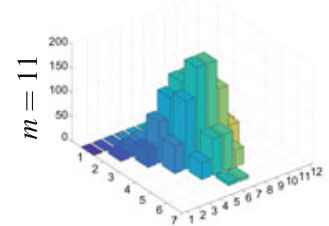

$\left(a_{2}\right)$

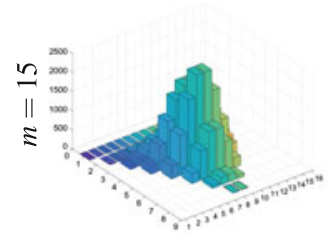

$\left(a_{3}\right)$

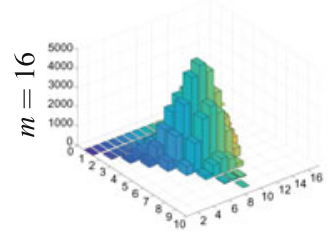

$\left(a_{4}\right)$

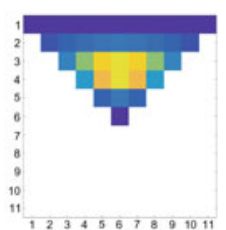

$\left(b_{1}\right)$

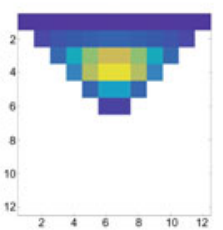

$\left(b_{2}\right)$

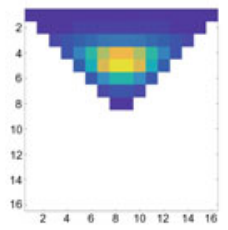

$\left(b_{3}\right)$

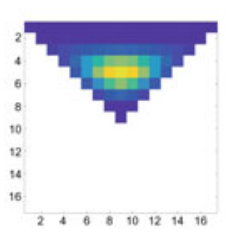

$\left(b_{4}\right)$

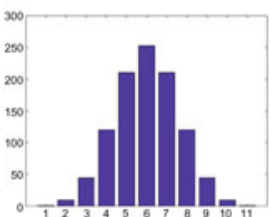

$\left(c_{1}\right)$

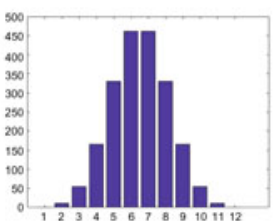

$\left(c_{2}\right)$

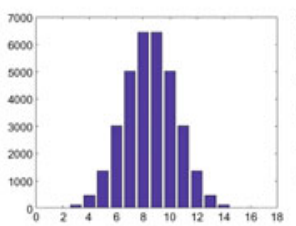

$\left(c_{3}\right)$

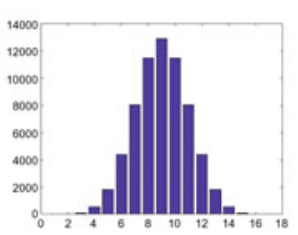

$\left(c_{4}\right)$

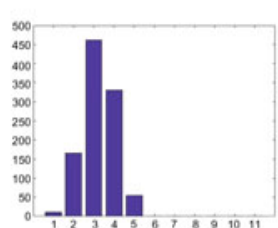

$\left(d_{1}\right)$

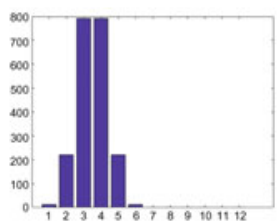

$\left(d_{2}\right)$

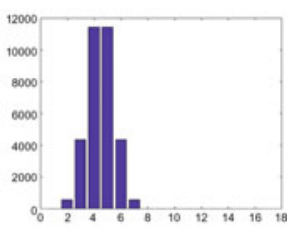

$\left(d_{3}\right)$

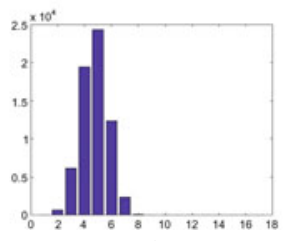

$\left(d_{4}\right)$

Fig. $2\{m-p\}\{p\} \quad$ maps: $\quad m=\{10,11,15,16\} ; \quad\left(a_{1}\right)-\left(d_{1}\right) m=10 ; \quad\left(a_{2}\right)-\left(d_{2}\right) m=11$; $\left(a_{3}\right)-\left(d_{3}\right) m=15 ;\left(a_{4}\right)-\left(d_{4}\right) m=16$

4, $8: 2,10: 0\}$, this can be described as $(x+y)^{n+l}=(x+y)^{n}(x+y)^{l}$ coefficient distributions that can be illustrated in Fig. $2\left\{\left\{\left(a_{0}\right)-\left(c_{0}\right)\right\}-\left\{\left(a_{5}\right)-\left(c_{5}\right)\right\}\right\}$ maps. 


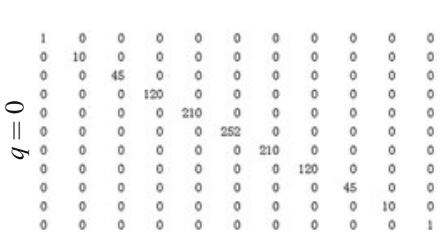

$\left(a_{0}\right)$

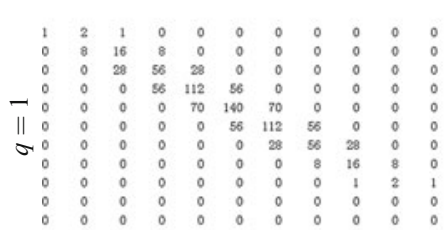

$\left(a_{1}\right)$

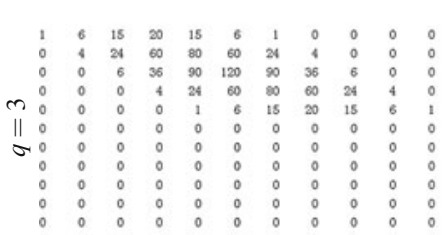

$\left(a_{2}\right)$

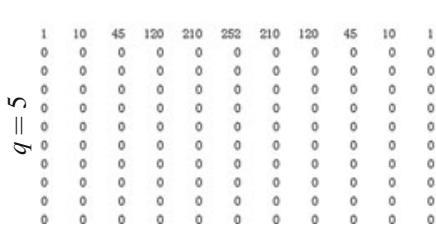

$\left(a_{3}\right)$

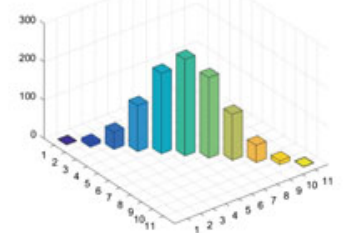

$\left(b_{0}\right)$

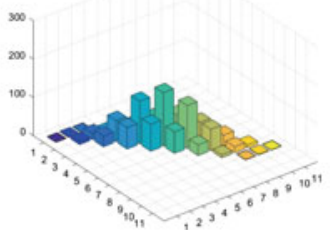

$\left(b_{1}\right)$

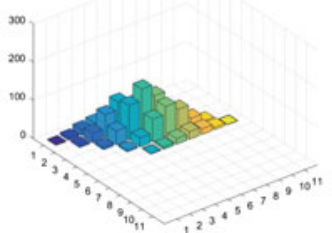

$\left(b_{2}\right)$

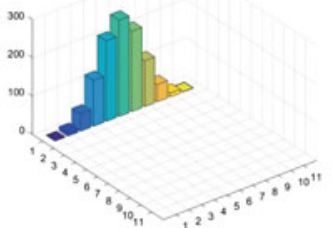

(b3)

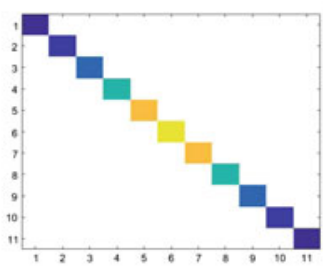

$\left(c_{0}\right)$

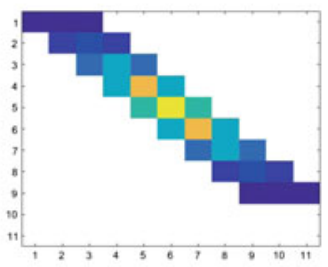

$\left(c_{1}\right)$

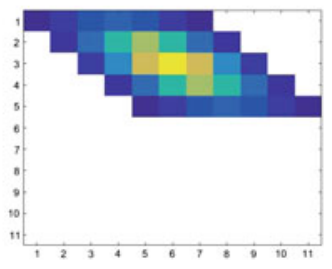

$\left(c_{2}\right)$

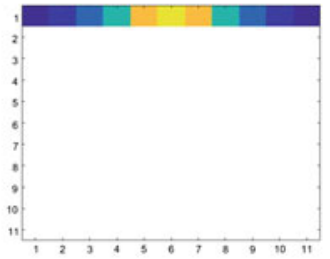

(c $\left.c_{3}\right)$

Fig. $3\{2 q\}\{m-2 q\}$ maps: $m=10 ; \quad\left(a_{0}\right)-\left(c_{0}\right) q=0 ; \quad\left(a_{1}\right)-\left(c_{1}\right) q=1 ; \quad\left(a_{2}\right)-\left(c_{2}\right) q=2$; $\left(a_{3}\right)-\left(c_{3}\right) q=3 ;\left(a_{4}\right)-\left(c_{4}\right) q=4 ;\left(a_{5}\right)-\left(c_{5}\right) q=5$ 
Fig. $4\{2 q\}\{m-2 q\}$

map: $m=10 ; 3 \mathrm{D}$ color map

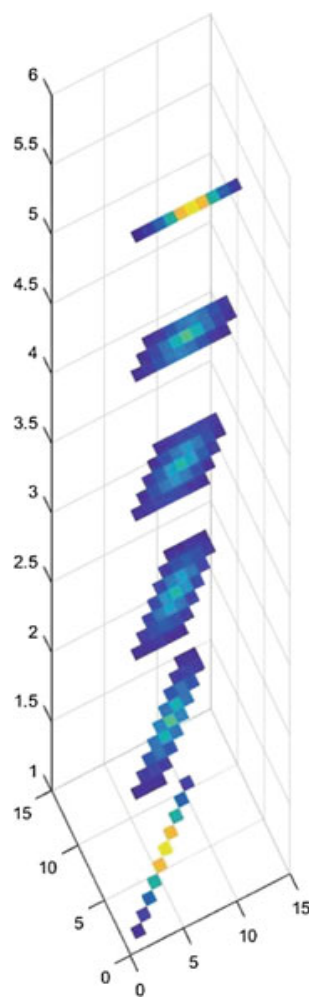

\section{Conclusion}

It is a new exploration to use elementary equation to illustrate relevant variant maps. Based on the described model and calculation, it is convenient to do various analysis and visualization. It is an initial step to check two invariants from Type B for four variant measures. Further explorations are required on five levels of 11 nontrivial invariants in Type B. From results in this chapter, distinct distributions are observed on the two selected invariants. Other nine invariants in Type B will be discussed in future papers (Fig. 4).

Acknowledgements The author would like to thank Yifeng Zheng and Kaiyu Yang for generating binomial coefficients in different conditions and Dr. Dennis Heim for correction of the chapter. 
Open Access This chapter is licensed under the terms of the Creative Commons Attribution 4.0 International License (http://creativecommons.org/licenses/by/4.0/), which permits use, sharing, adaptation, distribution and reproduction in any medium or format, as long as you give appropriate credit to the original author(s) and the source, provide a link to the Creative Commons license and indicate if changes were made.

The images or other third party material in this chapter are included in the chapter's Creative Commons license, unless indicated otherwise in a credit line to the material. If material is not included in the chapter's Creative Commons license and your intended use is not permitted by statutory regulation or exceeds the permitted use, you will need to obtain permission directly from the copyright holder.

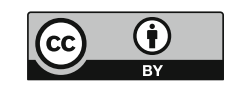

